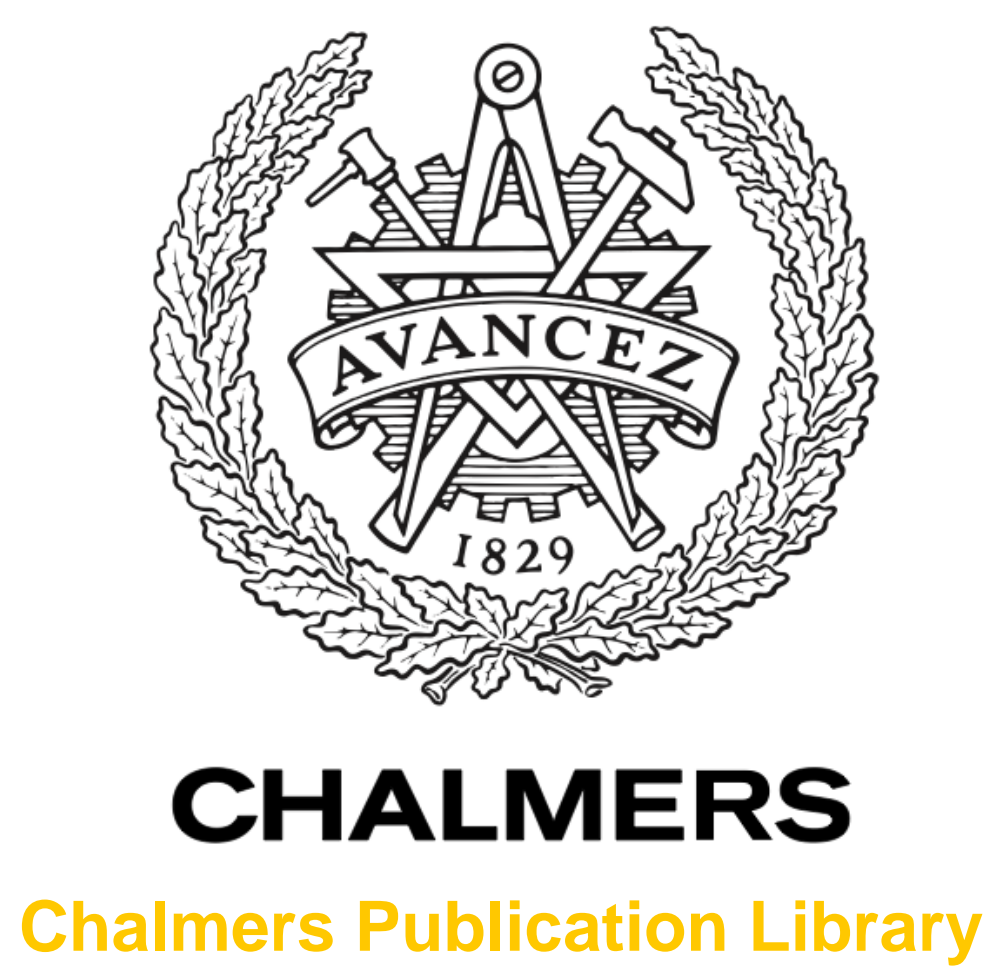

\title{
Carbon nanotube bolometers
}

This document has been downloaded from Chalmers Publication Library (CPL). It is the author's version of a work that was accepted for publication in:

\section{Applied Physics Letters (ISSN: 00036951)}

Citation for the published paper:

Tarasov, M. ; Svensson, J. ; Kuzmin, L. (2007) "Carbon nanotube bolometers". Applied

Physics Letters, vol. 90 pp. 163503.

http://dx.doi.org/10.1063/1.2722666

Downloaded from: http://publications.lib.chalmers.se/publication/63870

Notice: Changes introduced as a result of publishing processes such as copy-editing and formatting may not be reflected in this document. For a definitive version of this work, please refer to the published source. Please note that access to the published version might require a subscription. 


\title{
Carbon nanotube bolometers
}

\author{
M. Tarasov ${ }^{\text {a) }}$ \\ Institute of Radio Engineering and Electronics, 125009 Moscow, Russia \\ J. Svensson \\ Department of Physics, Göteborg University, SE-41296 Göteborg, Sweden \\ L. Kuzmin \\ Department of Microtechnology and Nanoscience, Chalmers University of Technology, SE-41296 Göteborg, \\ Sweden \\ E. E. B. Campbell \\ Department of Physics, Göteborg University, SE-41296 Göteborg, Sweden
}

(Received 23 January 2007; accepted 13 March 2007; published online 16 April 2007)

A cryogenic bolometer has been fabricated using a bundle of single-walled carbon nanotubes as absorber. A bolometric response was observed when the device was exposed to radiation at $110 \mathrm{GHz}$. The temperature response was $0.4 \mathrm{mV} / \mathrm{K}$, with an intrinsic electrical responsivity at low frequency up to $10^{9} \mathrm{~V} / \mathrm{W}$ and noise equivalent power of $3 \times 10^{-16} \mathrm{~W} / \mathrm{Hz}^{1 / 2}$ at $4.2 \mathrm{~K}$. The response is largest at input power levels of a few femtowatts and decreases inversely proportional to the input power. Low frequency noise shows a $1 / f$ dependence. (c) 2007 American Institute of Physics. [DOI: $10.1063 / 1.2722666]$

Recent breakthroughs in cosmology have stimulated the development of a generation of detectors for millimeter and submillimeter wavelengths. The detectors should be fast and sensitive to perform a fast survey of the sky for measurements of, e.g., cosmic background radiation. The advanced cameras developed for astronomy usually find applications also in terahertz imaging for security, medical, and biological applications. For these applications, it is required to have fast and sensitive bolometers working at relatively high temperatures. A bolometer with a carbon nanotube (CNT) is an attractive option for these applications.

It was predicted in Ref. 1 that superconductor-insulatornormalmetal-insulator-superconductor (SINIS) structures can be effective bolometers and electron coolers of normal metal absorbers, similar to conventional semiconductor Peltier elements. Experimental cooling from 300 down to $100 \mathrm{mK}$ in a SINIS structure was demonstrated in Ref. 2 and bolometric response up to $2 \mathrm{THz}$ in Ref. 3. The characteristics of such devices can be estimated using the relation for energy transfer between electrons and phonons, $P=\Sigma \nu\left(T_{e}^{5}\right.$ $\left.-T_{o}^{5}\right)$, or for electron temperature, $T_{e}=\left(T_{o}^{5}+P / \Sigma \nu\right)^{1 / 5}$, in which $\Sigma$ is a material parameter, $\nu$ is the absorber volume, and $T_{o}$ is the phonon temperature. According to these expressions, in order to increase the efficiency of warming by radiation and improve electron cooling, it is necessary to considerably reduce the volume of the absorber. In a conventional bolometer with a thin-film absorber fabricated using e-beam lithography, ${ }^{3}$ the volume is typically $10 \times 0.2$ $\times 0.05=0.1 \mu \mathrm{m}^{3}$. It should be possible to reduce the volume of the absorber by replacing the metal with a carbon nanotube. In this way the volume could be reduced to 10 $\times(0.0005)^{2} \times 3.14 \approx 10^{-5} \mu \mathrm{m}^{3}$, that is, four orders of magnitude smaller for an absorber of the same length.

Recently, a bolometric response to near-infrared radiation was reported for suspended macroscopic films of en-

\footnotetext{
${ }^{a)}$ Electronic mail: mikhail.tarasov@mc2.chalmers.se
}

tangled single-walled nanotubes. ${ }^{4}$ In that work, the nanotube samples had dimensions of $3.5 \times 0.5 \mathrm{~mm}^{2}$ with a thickness in the range of $40 \mathrm{~nm}-1 \mu \mathrm{m}$. No bolometric response could be detected for nonsuspended nanotube films. In this letter, we report the results of a proof-of-principle experiment, where we show a strong bolometric response of deposited nanotube bundles to microwave radiation. This provides encouragement for the development of sensitive cold electron bolometers based on individual carbon nanotubes as the absorber, where one could expect considerably enhanced electron cooling to temperatures below $100 \mathrm{mK}$.

Our samples were fabricated on $7 \times 7 \mathrm{~mm}^{2}$ oxidized $n$-doped silicon wafers. Ti/Au electrodes were first patterned using photolithography. An ac voltage is applied between the electrodes for dielectrophoretic deposition of single-walled nanotubes dispersed in $\mathrm{H}_{2} \mathrm{O}$ with 4 wt \% of sodium dodecyl sulphate. A relatively high nanotube concentration was deliberately used to deposit bundles of thousands of nanotubes rather than individual nanotubes between the electrode pairs. The voltage applied was $20 \mathrm{~V}$ peak to peak at a frequency of $13 \mathrm{MHz}$. After the nanotube deposition, four aluminum electrodes were deposited on top of the nanotube bundles. A typical device is shown in Fig. 1. The deposited nanotubes are expected to have a relatively high content of metallic nanotubes, ${ }^{5}$ but semiconducting nanotubes will also be present in the deposited bundles. Arrays of deposited nanotubes were preferred for these initial experiments instead of individual nanotubes, in order to reduce the resistance of the sample and enhance the response. The resistance of such samples at a temperature of $4 \mathrm{~K}$ was down to a few kiloOhms. The main part of this resistance seems to be due to the contact resistance.

The voltage response of such a CNT array to microwave radiation $(110 \mathrm{GHz})$ was measured. The source of radiation was an impact ionization avalanche transit-time diode with an output power of about $10 \mathrm{~mW}$. The signal was modulated with a mechanical chopper and attenuated with an ECCOSORB absorber. The radiation illuminated the CNT 


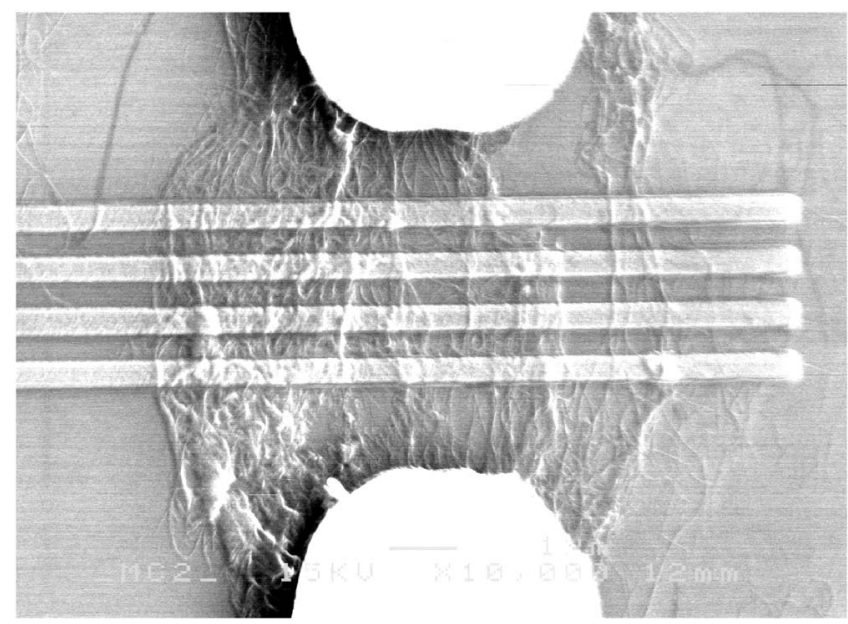

FIG. 1. Carbon nanotube bundles deposited across the top and bottom electrodes using ac dielectrophoresis. Four Al electrodes were patterned on top of the deposited nanotubes.

sample through a quasioptical window and cold IR filters. The sample was mounted on the flat side of an silicon extended hyperhemisphere silicon lens, $12.6 \mathrm{~mm}$ in diameter. The back side of the Si substrate was turned toward the silicon lens. The two of four outer Al electrodes (see Fig. 1) were dc biased and the voltage response on the two inner electrodes was lock-in detected, while the electrodes used for dielectrophoresis were kept unconnected. The $I-V$ characteristics of the sample at $4.2 \mathrm{~K}$, in the absence of microwave radiation, are shown in Fig. 2 (left hand axis) for a temperature of $4.2 \mathrm{~K}$. The microwave response for a dc bias from -100 to $+100 \mathrm{nA}$ is shown on the same plot (right hand axis), demonstrating an output signal of over $50 \mu \mathrm{V}$. The behavior looks very similar to the $I-V$ curve and the response of a conventional SINIS bolometer with an absorber made of a normal metal thin-film strip. ${ }^{3}$ The difference is that in a SINIS bolometer, the nonlinearity originates from the superconductor energy gap of the SIN junction, and in the CNT junction, the nonlinearity is due to other effects such as Schottky-type junctions or Coulomb blockade.

The temperature dependence of the voltage on the same sample was measured at a bias current of $0.3 \mu \mathrm{A}$ (see Fig. $3)$. From this dependence we can calculate a temperature response $d V / d T=0.4 \mathrm{mV} / \mathrm{K}$ for $T<50 \mathrm{~K}$ that is close to the

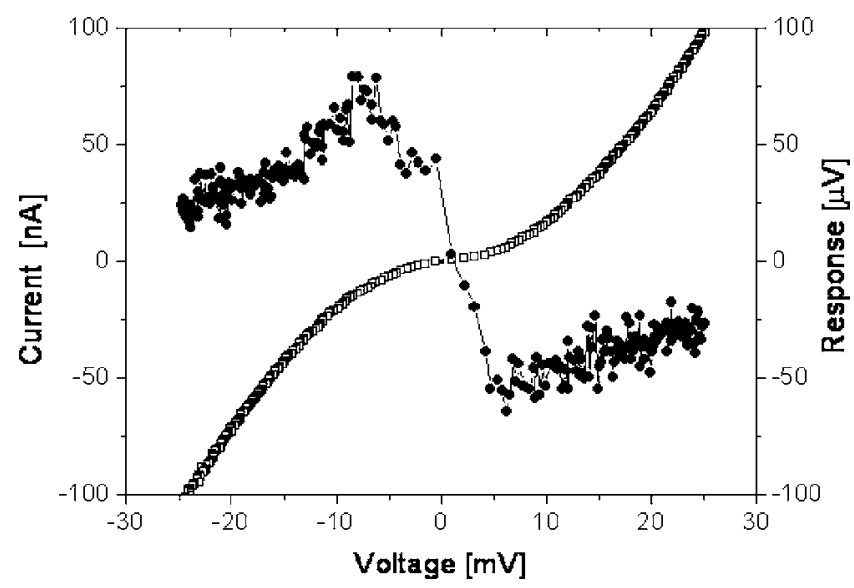

FIG. 2. $I$ - $V$ curve (left hand axis) measured between inner electrodes and voltage response of the nanotube device shown in Fig. 1, exposed to $110 \mathrm{GHz}$ radiation. Measurements were performed at $4.2 \mathrm{~K}$.

Downloaded 24 Apr 2007 to 129.16.137.132. Redistribution subject

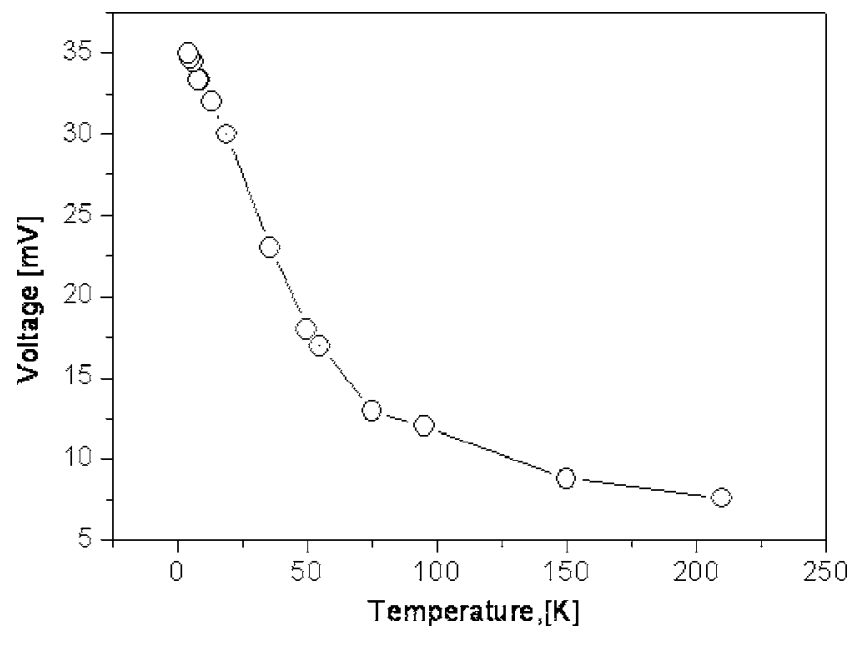

FIG. 3. Temperature dependence of the response voltage for a bias current of $0.3 \mu \mathrm{A}$.

temperature response of the metallic thin-film SINIS bolometer in Ref. $3(1.7 \mathrm{mV} / \mathrm{K})$.

Due to a huge impedance mismatch $(100 \Omega-300 \mathrm{k} \Omega)$ and high losses in the quasioptical beamguide, the incident power in the microwave measurements that is absorbed in the whole bundle can be very roughly estimated to be below $1 \mathrm{nW}$. To improve the accuracy of the responsivity measurements, we performed much more accurate ac measurements. The ac response was measured by applying an ac signal to the outer pair of the four electrodes and using the inner electrode pair as the detector at varying dc bias. The results are shown in Fig. 4 for an input power of $2.4 \mathrm{fW}$. There is a strong dependence of the response on the input signal power (Fig. 5). One can see that already for powers greater than a few femtowatts, the response decreases inversely proportional to the applied power.

To estimate the sensitivity, the noise of the device was also measured. It shows clear $1 / f$ dependence (Fig. 6). From these measurements it is possible to deduce the noise equivalent power (NEP) by dividing the noise by the response. The best $\mathrm{NEP}=3 \times 10^{-16} \mathrm{~W} / \mathrm{Hz}^{1 / 2}$ was measured at an input amplitude of $2.4 \mathrm{fW}$ and an input frequency of $700 \mathrm{~Hz}$, at

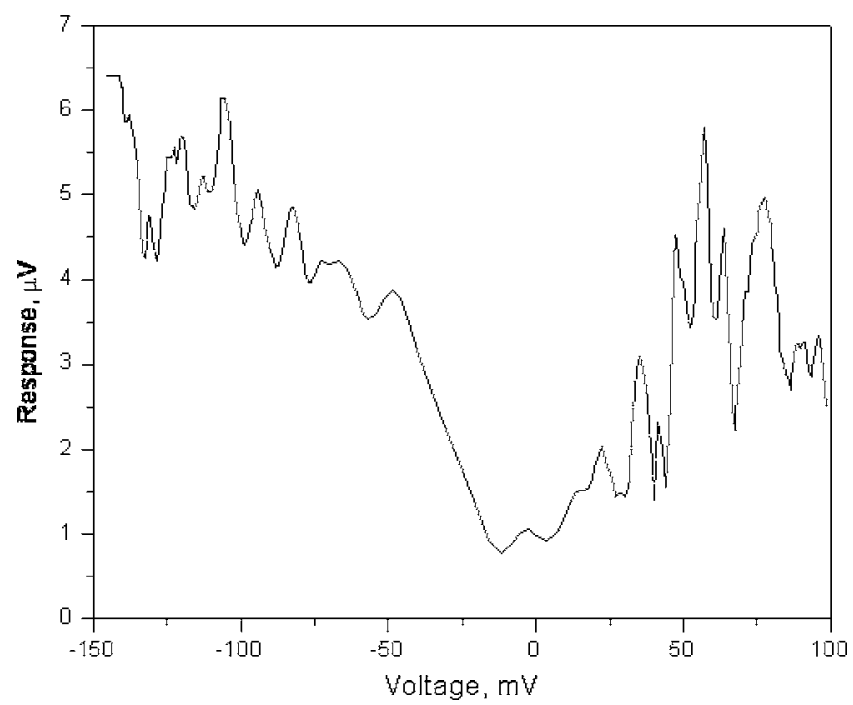

FIG. 4. ac response for input probe power of $2.4 \mathrm{fW}$. ac voltage applied across outer electrode pair; response measured across inner electrode pair. AIP license or copyright, see http://apl.aip.org/apl/copyright.jsp 


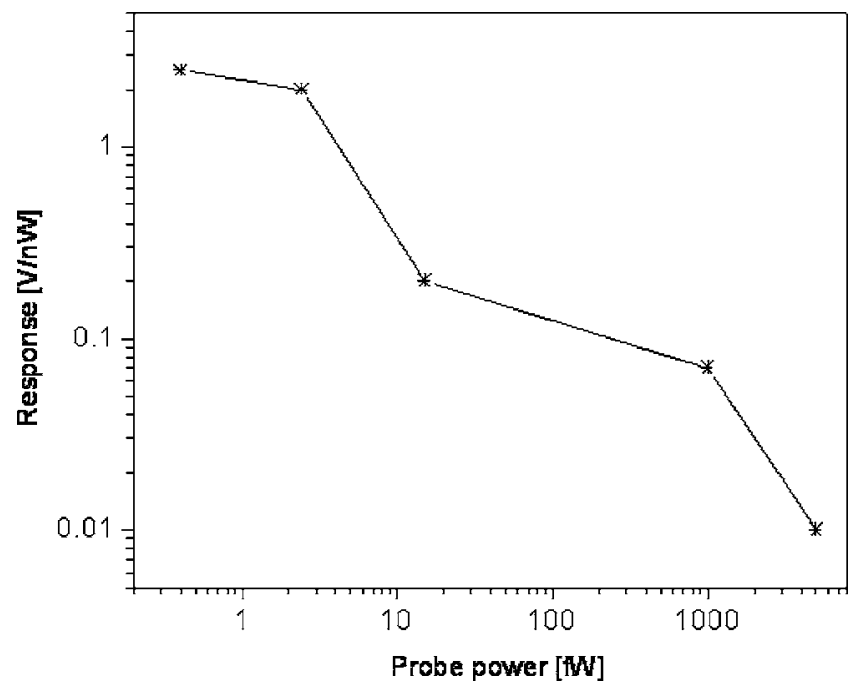

FIG. 5. Dependence of response on input probe power.

which the noise impact from the readout amplifier was the lowest.

We have demonstrated that carbon nanotubes can operate as bolometric detectors at microwave frequencies. Due to the small diameter of carbon nanotubes, the junction capaci-

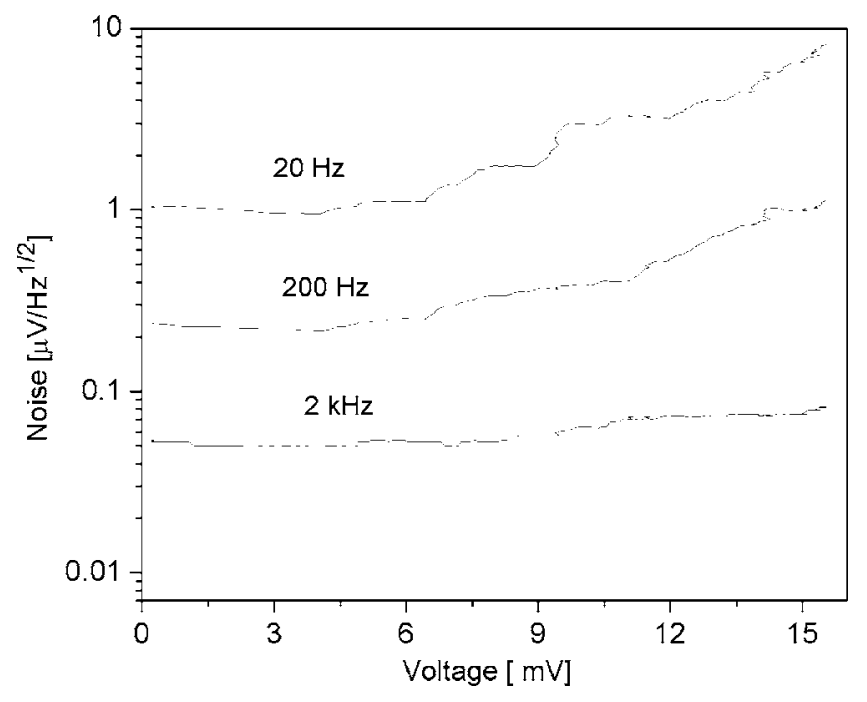

FIG. 6. Frequency dependence of noise in semilogarithmic plot. tance can be significantly smaller than for normal Schottky detectors, which could push the detection frequency cutoff to beyond $30 \mathrm{THz} .{ }^{6,7}$ Besides bolometric detectors, other microwave applications of CNTs such as mixers, ${ }^{8}$ microwave field-effect transistors, ${ }^{9}$ and direct and heterodyne detectors ${ }^{10}$ have been realized. The NEP obtained in our work is better than what has been achieved with other direct detector device at an operating temperature of $4.2 \mathrm{~K}$ (see Ref. 11). However, it is important to emphasize that the measured NEP is for intrinsic electrical responsivity, while similar optical characteristics are many orders of magnitude worse. The fabrication technology should be further developed to improve impedance matching and quality of CNTs. The small volume of CNTs also makes them promising materials for the construction of cold electron bolometers formed from superconductor-insulator-normal metal structures. ${ }^{1}$ Electron refrigeration from 360 down to $120 \mathrm{mK}$ was recently demonstrated in a superconductor-insulator-normal metal structure, ${ }^{2}$ and we expect to be able to improve this by using single CNTs or CNT bundles. However, for this purpose, the fabrication technology of the nanotube contacts will have to be improved.

This work was supported by the Swedish Institute, Vetenskapsrådet, K \& A Wallenberg Foundation, and Grant Nos. PGLSS 7812.2006.2, and RFBR 05-02-19650.

${ }^{1}$ L. Kuzmin, I. Devyatov, and D. Golubev, Proc. SPIE 3465, 193 (1998).

${ }^{2}$ L. Kuzmin, I. Agulo, M. Fominsky, A. Savin, and M. Tarasov, Supercond. Sci. Technol. 17, 400 (2004).

${ }^{3}$ M. Tarasov, L. Kuzmin, E. Stepantsov, I. Agulo, A. Kalabukhov, Z. Ivanov, and T. Claeson, JETP Lett. 79, 298 (2004).

${ }^{4}$ M. Itkis, F. Borondics, A. Yu, and R. Haddon, Science 312, 413 (2006).

${ }^{5}$ D. S. Lee, D. W. Kim, H. S. Kim, S. W. Lee, S. H. Jhang, Y. W. Park, and

E. E. B. Campbell, Appl. Phys. A: Mater. Sci. Process. 80, 5 (2005).

${ }^{6}$ D. Tsang and S. Schwartz, Appl. Phys. Lett. 30, 263 (1977).

${ }^{7}$ H. M. Manohara, E. W. Wong, E. Schlecht, B. D. Hunt, and P. H. Siegel, Nano Lett. 5, 1469 (2005).

${ }^{8}$ S. Rosenblatt, H. Lin, V. Sazonova, S. Tiwari, and P. L. McEuen, Appl. Phys. Lett. 87, 153111 (2005).

${ }^{9}$ A. A. Pesetski, J. E. Baumgardner, E. Folk, J. Przybysz, J. D. Adam, and H. Zhang, Appl. Phys. Lett. 88, 113103 (2006).

${ }^{10}$ F. Rodriguez-Morales, R. Zannoni, J. Nicholson, M. Fischetti, K. S. Yngvesson, and J. Appenzeller, Appl. Phys. Lett. 89, 083502 (2006).

${ }^{11}$ A. Luukanen, E. N. Grossman, A. J. Miller, P. Helistö, J. S. Penttilä, H. Sipola, and H. Seppä, IEEE Microw. Wirel. Compon. Lett. 16, 464 (2006). 